\title{
The Relationship between the Dental Occlusion, Upper Cervical spine and Temporomandibular Joints prior to and following TENS treatment in thirty six patients suffering from Temporomandibular Disorder
}

\author{
Norman Randall Thomas ${ }^{1 *}$, Trystan David Thomas ${ }^{2}$ and Norman Richard Thomas ${ }^{3}$ \\ ${ }^{1}$ Faculty of Medicine and Dentistry, University of Alberta; Canada, Past Director Neuromuscular Research, Las Vegas Institute of Advanced Dental Studies, \\ Chancellor of ICCMO, USA \\ ${ }^{2}$ Neuromusc.Res. Assoc Las Vegas Inst. Advanced Dental Studies, USA \\ ${ }^{3}$ Case Western Reserve U, Las Vegas Inst, Advanced Dental Studies, USA
}

\begin{abstract}
Transcutaneous electrostimulation (TENS) of the Vth,VIIth and XI cranial nerves produces relaxation of the masticatory, facial and neck muscles by antidromic relaxation and hyperpolarisation of the alpha gamma motorneurone of the jaw elevator and depressor muscles of the head, jaw and neck via the mesencephalic proprioceptor nucleus of the trigeminal nerve. This results in concentricity and alignment of the atlanto occipital A/O,atlanto axial A/A and C3/C4 vertebrae in part previously described by Thomas 1990,Raman 2010 and Fujii and Mitani 1973.
\end{abstract}

\section{Introduction}

This paper is a rewrite of a prior LVIADS Visions Jan-Apr 2009 paper including research paper given at the International Anatomy Congress St Petersburg Russia 1970 [12]

Cooperman, et al. [1-4] noted from the Louchheim skull collection that the horizontal Plane (HIP) through the hamular notches $(\mathrm{H})$ and the incisal papilla (IP) defines the plane of the basal bone of the maxilla which extends dorsally through the atlanto occipital (AO) joint of the upper cervical vertebral joints. On this HIP the alveolar bone develops to support the maxillary teeth on an occlusal plane with the mandibular teeth which in turn extends through the atlanto axial joint $\mathrm{C} 1 / \mathrm{C} 2$ Figure 1. We understand from the work of Harvold, et al. [5] that this occlusal plane may be corrupted by mouth breathing if the nasal airway is obstructed by nasal plugs or by hypertrophic nasopharyngeal adenoids. Also, the vertical dimension of occlusion may be limited when the force of dental eruption is retarded by failure of crosslinkage of the collagen in the periodontal ligament which among supportive properties serve to connect the teeth to the alveolar bone [6,7]. Thus, the occlusal plane may be further corrupted by concomitant tongue thrust swallow resulting from oral restriction from hypertrophic palatal tonsils or from an enlarged tongue developing from obesity or other pathology.

It will be noted that while the posterior extension of the HIP plane bisects the atlanto-occipital $(\mathrm{A} / \mathrm{O})$ joint the parallel occlusal plane should bisect the atlantoaxial joint space from a physiological occlusion as in Bimler Cephalometric Figure 2 [8]. The lateral skull x-ray Figure 3 which is not a cephalometric radiograph is given for comparison. As pointed out above the eruption process may be impeded in association with an overclosure of the vertical dimension of occlusion derived from a variety of dental malocclusions such as Angle's Class II Div 1 where the mandibular dental arch is retruded with respect to the maxillary arch Figure 4 . The pharyngeal airway may be restricted in obstructive sleep apnoea OSA when voluntary control of the airway is lost during sleep. This restriction may collapse down to a diameter of $2 \mathrm{~mm}$ from a normal range of $12 \mathrm{~mm}$ at the level of the hyoid bone at intervertebral space C3/C4 from kyphotic postural changes described as forward head posture when the upper spine is anteriorized while the head is extended dorsally to maintain the optic gaze. In the Bimler Cephalometric tracing of a healthy occlusion Figure 2 it should be observed that the occlusal plane forms a tangent to the Curve of Spee with its centrum masticale at its centre in the Falx Cerebri from which a perpendicular is dropped to the tangent of the occlusal plane bisects the long axis of the first premolar tooth. The Curve of Spee is a sphere which follows the lingual cusps and cinguli of the dentition which also delineates the Curve of Wilson in the transverse plane. The Curves of Spee and Wilson with their common centre at the centrum masticale (CM) extends a trajectory to the temporomandibular ligament which forms the axis of swing of the condyle and mandible as they arc forwards along the occlusion following the Curve of Spee. Note that in the ideal occlusion the temporomandibular ligament subtends an angle of thirty degrees

${ }^{\star}$ Correspondence to: Norman Randall Thomas, BDS (Hons.); MB.BSc. (Hons Physiol; Anat; Biochem.); PhD. U.Bris; FRCD.C; Cert Oral Path and Med. (MCV USA) FADI; MICCMO; DAAPM. Emeritus Professor, Faculty of Medicine and Dentistry, University of Alberta; Canada, Past Director Neuromuscular Research, Las Vegas Institute of Advanced Dental Studies; Chancellor of ICCMO, USA, E-mail: nthomas95@shaw.ca

Received: January 08, 2019; Accepted: January 15, 2019; Published: January 18, 2019 
Thomas NR (2019) The Relationship between the Dental Occlusion, Upper Cervical spine and Temporomandibular Joints prior to and following TENS treatment in thirty six patients suffering from Temporomandibular Disorder

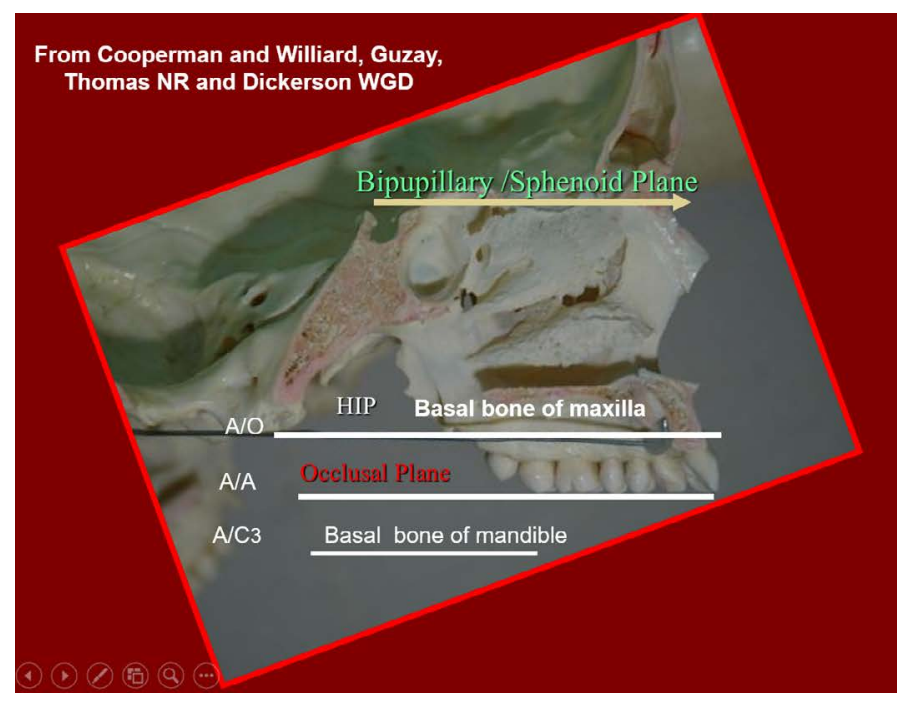

Figure 1. Maxillary teeth on an occlusal plane with the mandibular teeth

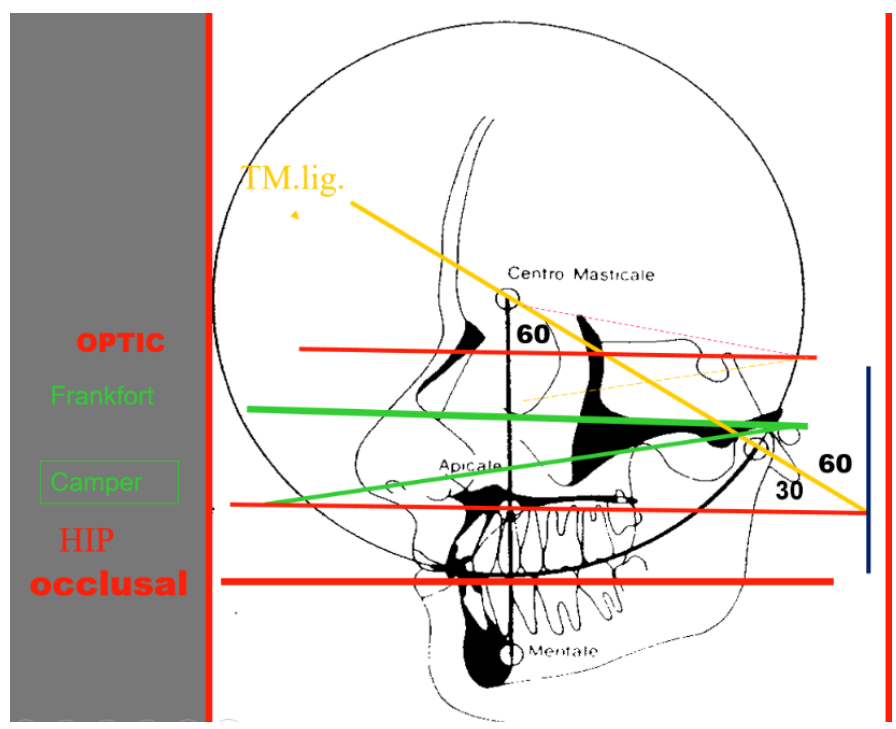

Figure 2. HIP plane bisects the atlanto-occipital (A/O) joint

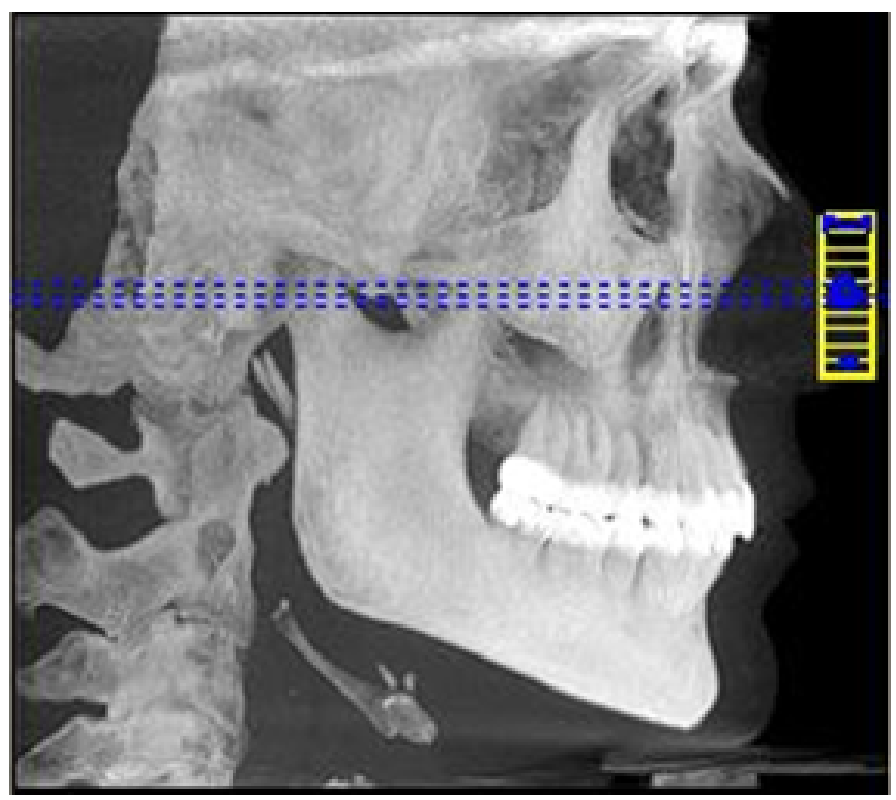

Figure 3. The lateral skull $\mathrm{x}$-rays 


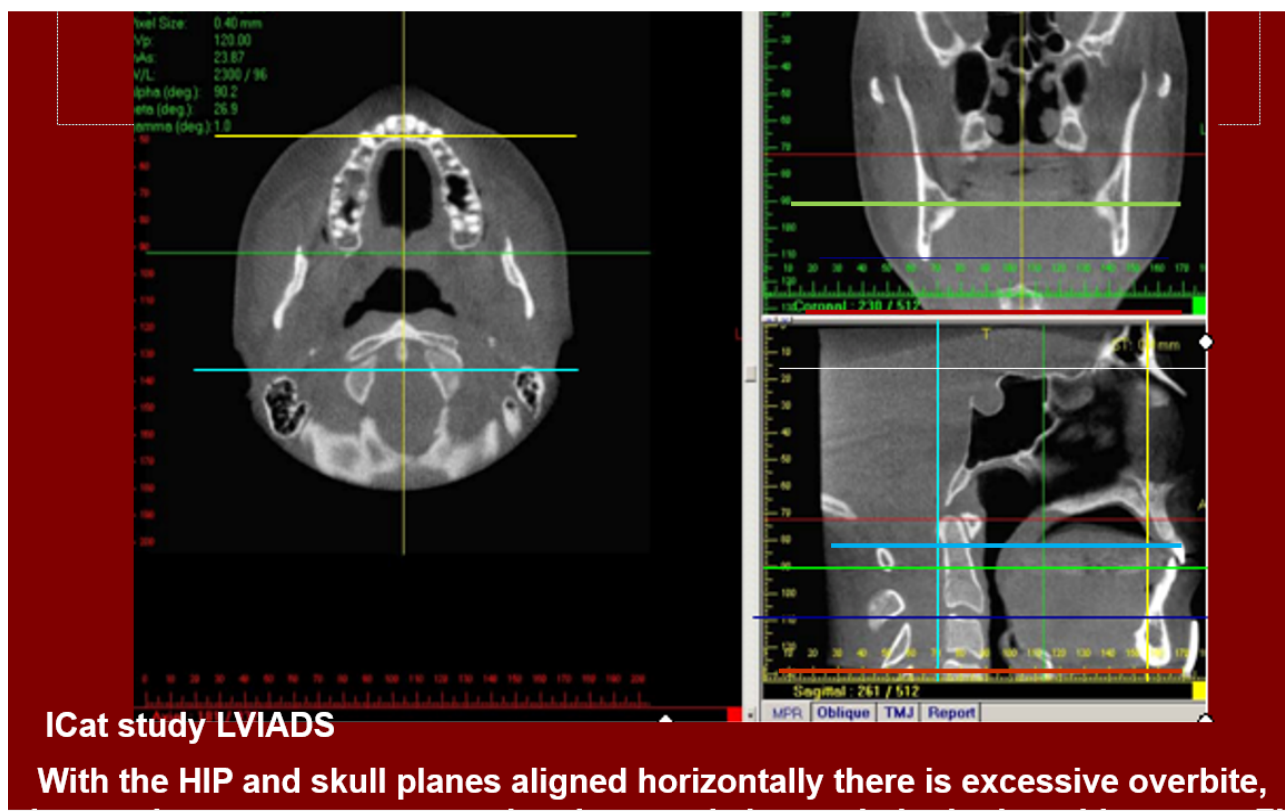

Figure 4. HIP and skull planes alignment

to the HIP as it passes through the temporomandibular joint (TMJ) to reach the centre of the motion of jaw function as defined by Guzay in the Quadrant Theory [9]. In Figure 2 it is seen that the tangent to the occlusal plane is parallel to the optic or pupillary plane and the also to the sphenoid base of the skull. Failure to understand the relationship of the maxillary lingual cusps and cinguli to the Curves of Spee and Wilson as well as to the occlusal plane, bipupillary plane, and HIP and $\mathrm{A} / \mathrm{O}$ joints has led to improper mounting of the maxillary dental casts on the horizontal stage of the occlusal evaluation articulator as illustrated in Figure 5. Arbitrary use of the Fox Plane in violation of Wolff's law relating stress and strain in regards to the morphological and physiological properties of the occlusal system is known to lead to muscle fatigue.

Figure 6 presents the more common right tilt 'stick men' following primary stimuli that are starred as descending and ascending varieties respective of postural anomalies (the uncommon left tilt, a complete reversal is not shown in this paper).It is evident that there is reciprocity between the craniomandibular, ocular, cervical and pelvic planes which are not strictly normal (parallel) to the gravitational field. The two examples on the left of the figure are due to a left functional short leg syndrome derived from descending and ascending stimuli respectively as opposed to the true short leg syndrome on the extreme right. These stick men describe the postural changes seen in patients during maintained static posture as well as during function as in chewing, swallowing, optic and shoulder posturing. This is directed at maintaining posture and the horizontal gaze which, in failure to conform to the gravitational field, results in descending anti parallelism between head and shoulder planes when the occlusal plane and skull base are not normal to the gravitational field (Thomas NR 1986 at the fifth international meeting of ICCMO) later published by Thomas NR, et al. [10]. Conversely parallel tilts of the head, shoulder and pelvic planes follow postural disturbances that arise from any point of injury caudal to the hyoid joints $\mathrm{C} 3 / \mathrm{C} 4$ joints and hence the occlusal plane anomaly is designated ascending parallelism which secondarily unbalances the occlusion with resulting neuromuscular dysfunction. Zafar, et al. [11] using a wireless optoelectronic system for three dimensional movement of natural head motion during jaw opening and closure which demonstrates reciprocity between head posture and jaw function. A consistent finding was parallel and coordinated

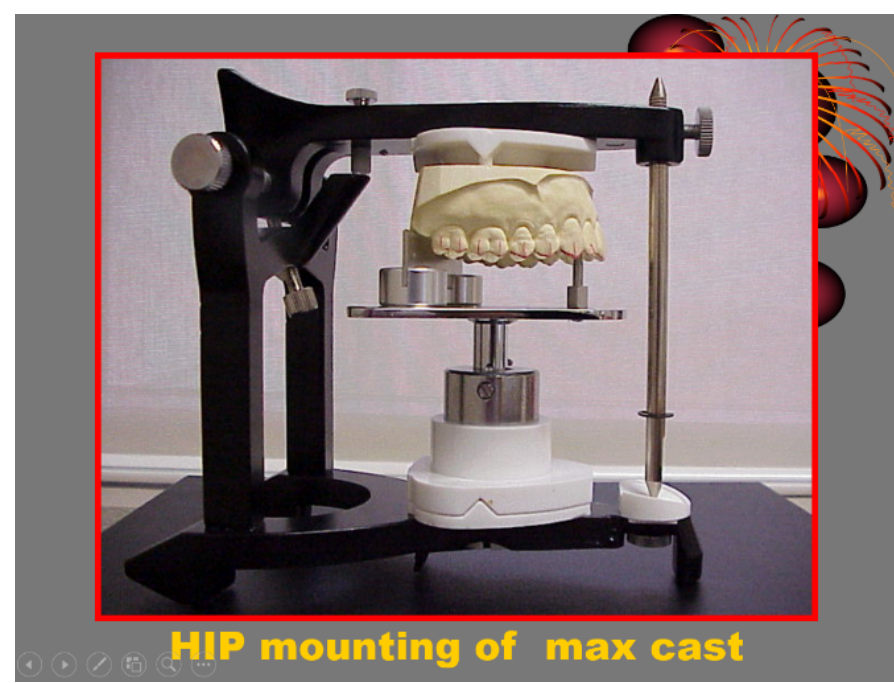

Figure 5. HIP mounting of max cast

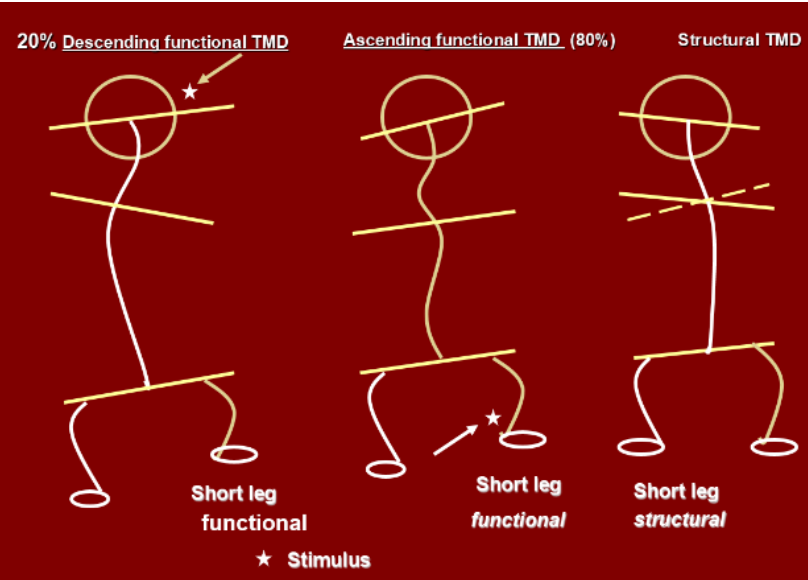

Thomas N.R 1986 ICCMO $5^{\text {th }}$ International Meeting Hawaii (later published in Anthology): Reflex Postural Figures due to 'TOP BLOCK' Mechanisms

Figure 6. Reflex postural figures due to TOP BLOCK mechanisms 
Thomas NR (2019) The Relationship between the Dental Occlusion, Upper Cervical spine and Temporomandibular Joints prior to and following TENS treatment in thirty six patients suffering from Temporomandibular Disorder

movements during fast and slow jaw opening-closing. It was noted that as the jaw opens the head extends posteriorly and vice versa in jaw closure. In Whiplash Disorder (WAD) or Acceleration Deceleration Disorder) decreased jaw movement accompanies decreased head and neck motion. Only anteroposterior motion defined as cranial pitch primarily occurs at the $\mathrm{A} / \mathrm{O}$ joints.

This indicates that the shape of the mandibular condyles conform to the shape of the occipital condyles and that protrusion of the TMJs may be cooperatively combined with retrusion of the occipital condyles. Given that condylar remodelling is usually more severe on one or other of the Joints retrusion of the mandible used in deriving centric relation occlusion clinically is inappropriately undertaken at most dental schools because it imposes unnecessary strain on the TMJs and cranio-occipital joints. Thus, such retrusion designated as 'seating' of the TMJs evokes retrusive subluxation of the occipital condyles. In Figures 7 and 8 it was demonstrated, as anticipated, that the wax impressions of TMJ and occipital condyles of fifty skulls at the Faculty of Dentistry at Edmonton perfectly conform to each other. For example, the medial pole of the TMJ condyle corresponds with the anterior pole of the occipital condyle. Thus, as the jaw is retruded by the clinician the skull tends towards retrusion which is opposite to cooperative physiological function of the TMJ and

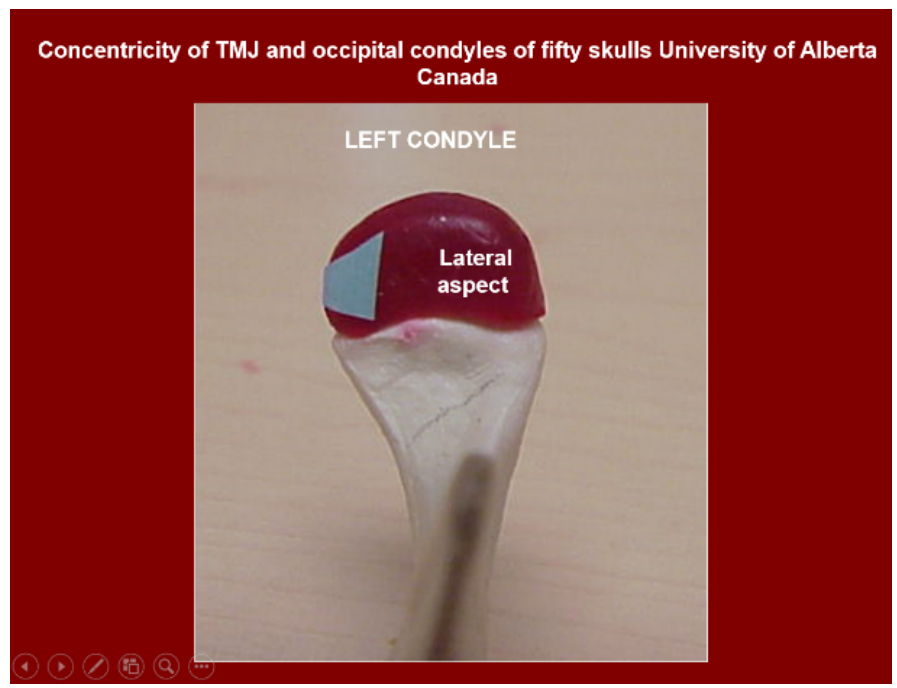

Figure 7. Concentricity of TMJ and occipital condyles of fifty skulls

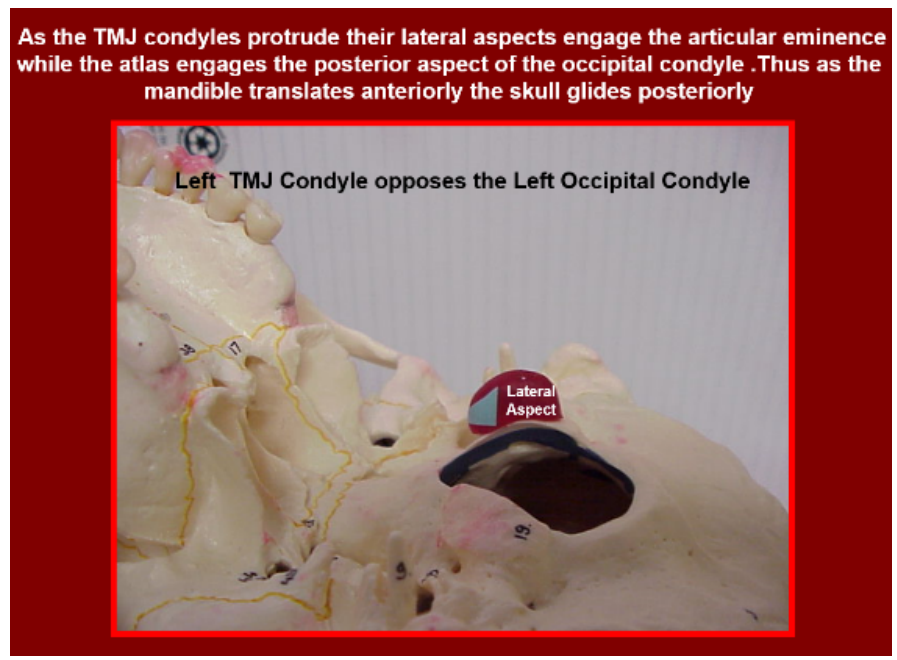

Figure 8. Left TMJ condyle opposes the Left occipital Condyle occipital joints during jaw opening and closing. This is one reason why neuromuscular dentists use transcutaneous relaxation of the cranial and masticatory musculature neither manipulatively restraining jaw or neck musculature. Thus as the skull glides directly over the occipital condyles it traverses the temporomandibular joints from lateral to medial poles. The significance of this is that as the neck flexes anteriorly as in forward head posture (FHP) the skull extends posteriorly in paradoxical fashion to maintain the horizontal gaze such that the jaw joints become posteriorly displaced into a Class II relationship and vice versa for Class III with mandibular protrusion. The neutral position of the head and neck accordingly assumes Class I relationship of the maxillo-mandibular processes where the superior pole of the TMJ condyle is opposed to the intermediate zone of the TMJ disc. Therefore, in conclusion the various skeleton-dental orthopaedic classifications of the maxillo -mandibular relationship I, II and III are intimately related to body posture. Furthermore because of this interactive relationship of the occipital and mandibular temporomandibular joint subluxations of the $\mathrm{A} / \mathrm{O}$ joints is accompanied by displacement of the mandibular condyles and vice versa. This was confirmed from studying the CBCT images of the cervical and temporomandibular condyles of 36 subjects suffering from TMD. Figures 9-14 are examples of pre/post TENS non-concentricity of TMJs and upper cervical spine vertebrae and concentricity post TENS muscle relaxation respectively. It is noted that in this sample $30 / 36$ subjects (83\%) exhibit posteriorly pitched crania corresponding to 54/72(75\%) posteriorly compressed temporomandibular joints of which $42 / 72(80 \%)$ TMJs have retrodiscal compression on the right side and 24/36 (80\%) A/Os are rolled to the right side. This relates to common handedness of the subjects which are predominantly right dominant eye, hand and side chewers. Thus $80 \%$ of subjects have posteriorly pitched skulls as in FHP with roll to the right. This number corresponds to the majority having an ascending body posture with parallelism of the head, neck and pelvic planes approximately $80 \%$ of all TMD /CMD craniomandibular dysfunctional patients while $20 \%$ are descending postures with anti-parallelism of the three planes. The descending types have primary TMD signs and symptoms which affects the posture rostro-caudally from above down. In the ascending type the body imbalance spreads upwards to affect the occlusion while in the descending type the effects of an abnormal occlusion spreads downwards to lead to postural compensations. These postural compensations arise from proprioceptor feed to the labyrinth balance organ in the inner ear with relay to the brainstem vestibular nucleus. Importantly there are major connections from the jaw and dental periodontal receptors to signal occlusal deviations to the same region of the brain known as the mesencephalic nucleus of the fifth nerve which has the only example of first order neurones entering the brain by a process known as neurobiotaxis. Such is the importance of the occlusion to total body neurophysiology. But the question emerges where the ascending mechanism begins and the descending mechanism end. Is it from a functionally short leg or from the rolled atlanto axial joints of the cervical spine? We do know that that the cervical tonic receptors play a significant role, but it is not clearly understood how this relates to the important mesencephalic trigeminal nucleus to which the proprioceptor information is relayed. This led us to examine the changes that occur in the cervical alterations that accompany the bite changes. Figure 14 shows the CBCT scans through the jaw joints and the upper cervical spine complex in thirty-six known TMD (36) patients prior to correction of the occlusion by removable /fixed prosthesis as in the rehabilitation of the edentulous and dentate patients respectively. It will be noted that the instantaneous axes of the upper cervical complex are non-concentric. Similarly, the jaw joints are more compressed on the right side $(3.2 \mathrm{~mm})$ corresponding to the decreased interval between the 
Thomas NR (2019) The Relationship between the Dental Occlusion, Upper Cervical spine and Temporomandibular Joints prior to and following TENS treatment in thirty six patients suffering from Temporomandibular Disorder

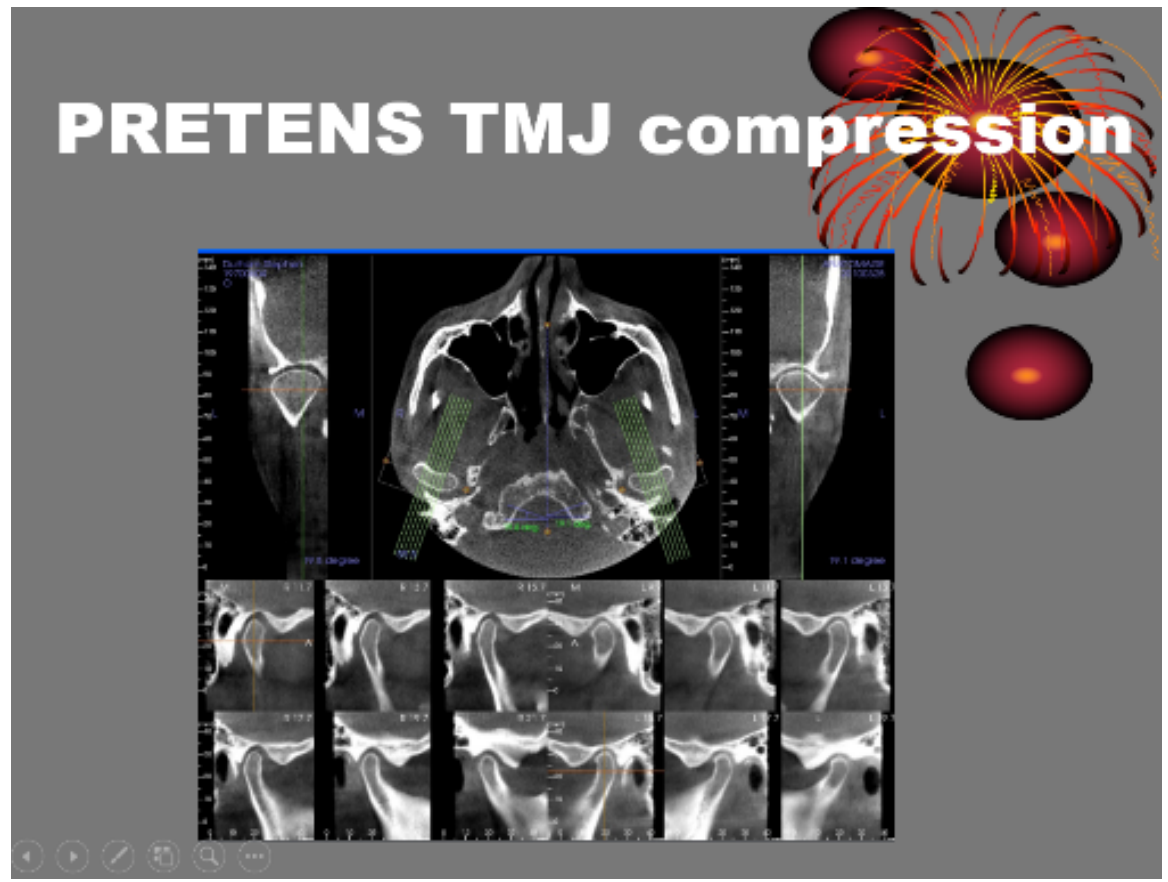

Figure 9. TMJ
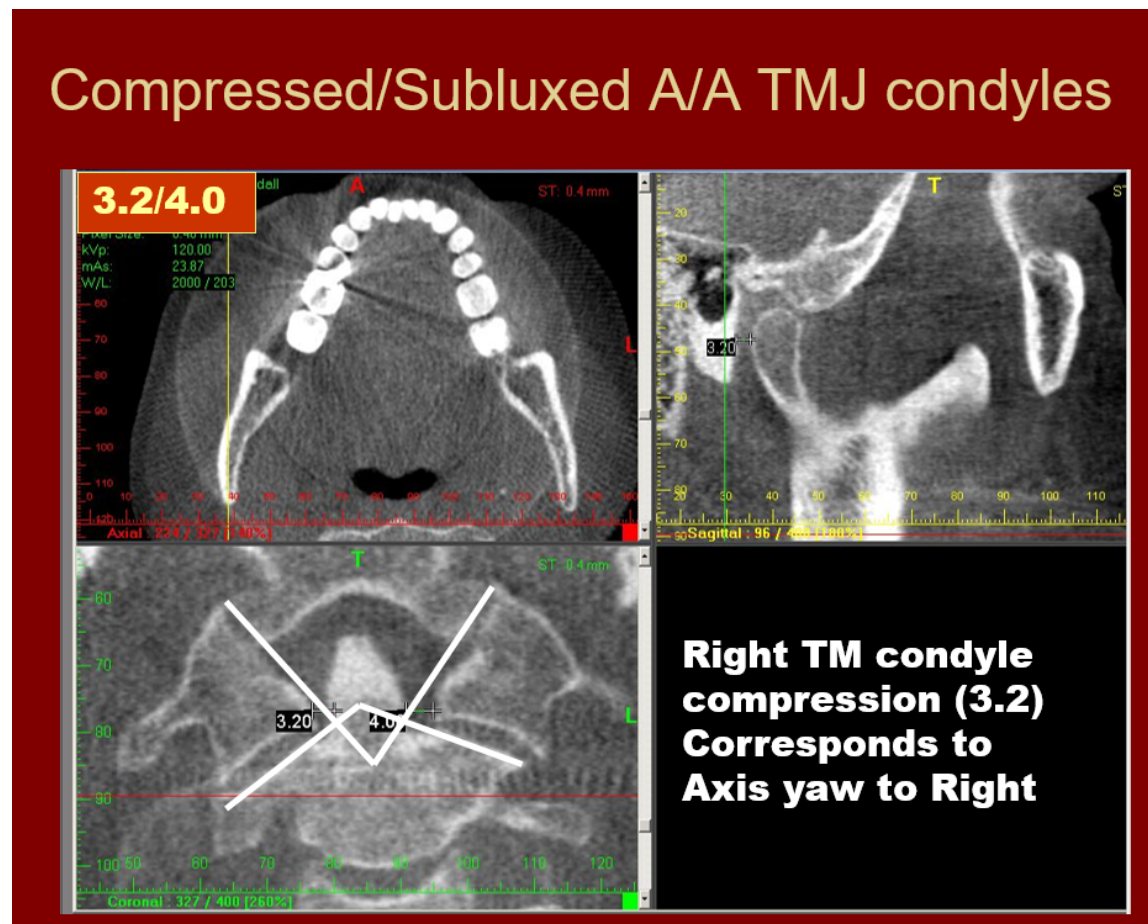

Figure 10. Compressed A/A TMJ condyles

odontoid process and the right atlas $(3.2 \mathrm{~mm})$ which is subluxed to the left side. This correlation was demonstrated for all subjects although the measurements may vary somewhat dependent upon the degree of TMJ remodelling. Following TENS relaxation of the neck and jaw musculature by antidromic activation and hyperpolarization of the trigeminal and cervical alpha and gamma neurones of the fifth and eleventh cranial nerves (Figures 15 and 16). The post relaxation CBCT images of the cervical vertebrae reveal concentricity of the atlanto- occipital and atlanto axial joints which line up with the midline of the vertebral column (Figure 13). In it is noted that the instantaneous centre for the atlanto axial joint is found centred at the intervertebral disc between $\mathrm{C} 3$ and $\mathrm{C} 4$ in the post TENS relaxed musculature supplied by the trigeminal and spinal accessory cranial nerves. The disc between $\mathrm{C} 3$ and $\mathrm{C} 4$ is found at the level of the hyoid bone which gives origin and insertion to the jaw openers and hence the anterior neck alignment which is markedly displaced in the pre TENS relaxed condition (Figure 
Thomas NR (2019) The Relationship between the Dental Occlusion, Upper Cervical spine and Temporomandibular Joints prior to and following TENS treatment in thirty six patients suffering from Temporomandibular Disorder

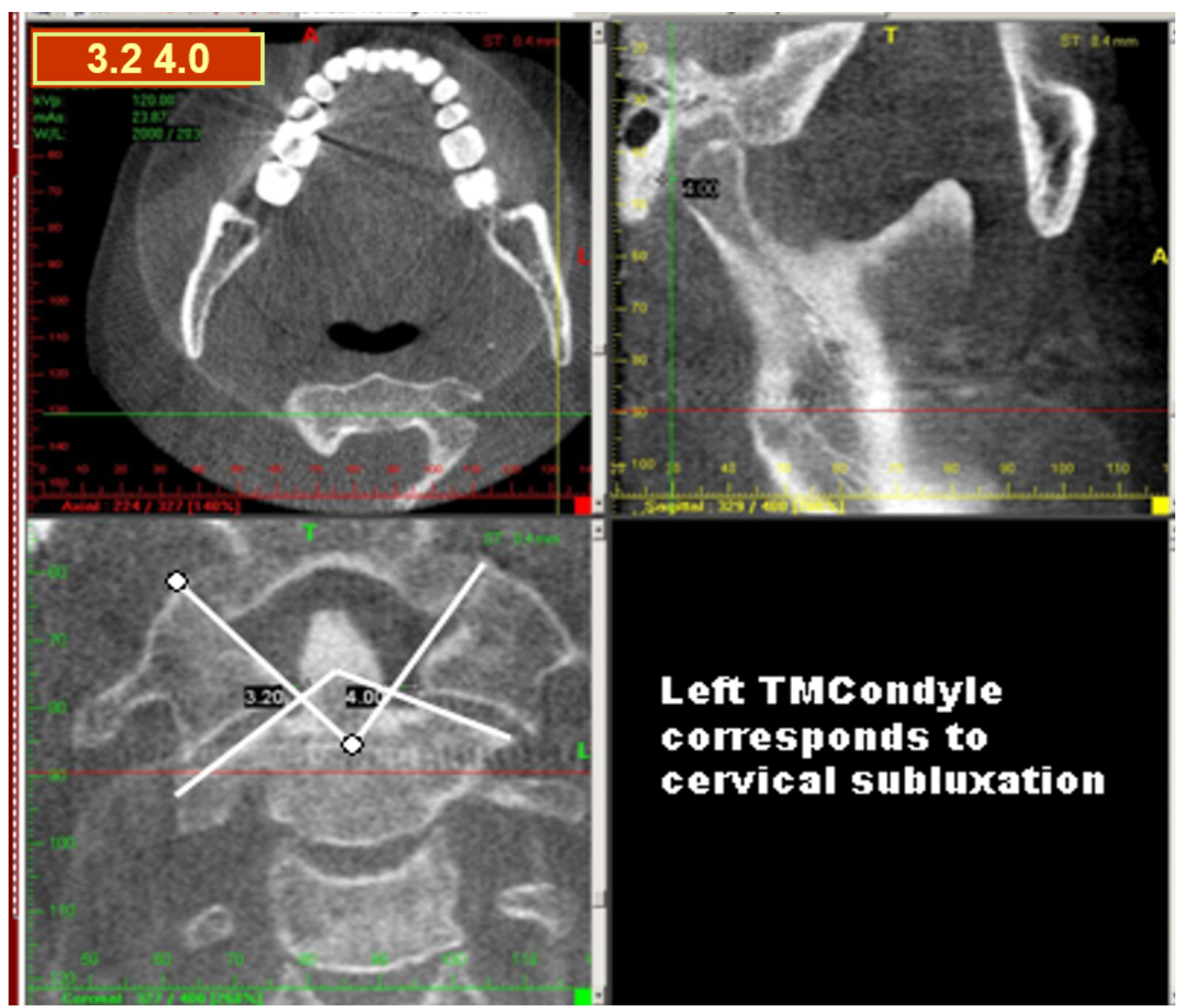

Figure 11. Left TMCondyle correspondence to cervical subluxation

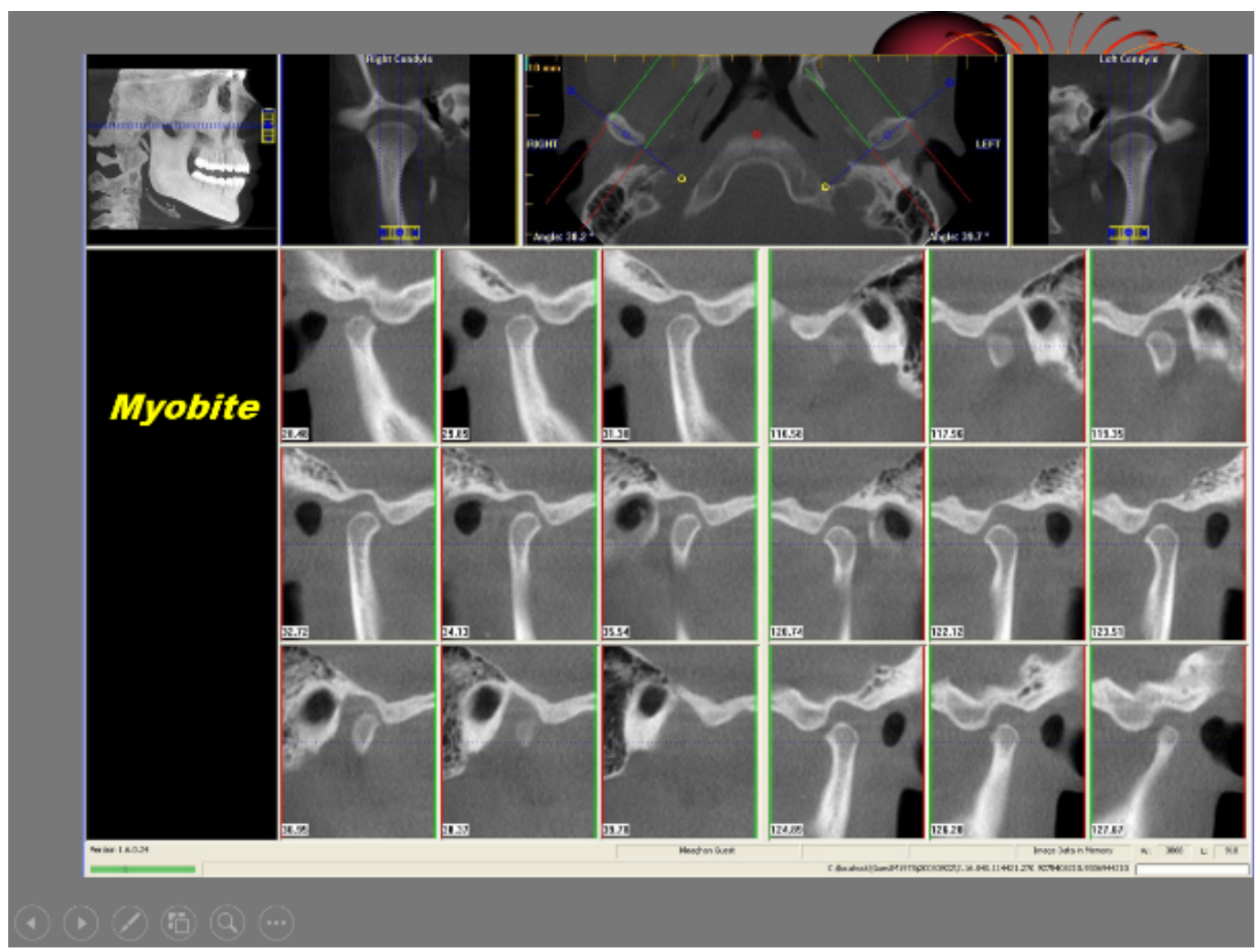

Figure 12. Myobite 
Thomas NR (2019) The Relationship between the Dental Occlusion, Upper Cervical spine and Temporomandibular Joints prior to and following TENS treatment in thirty six patients suffering from Temporomandibular Disorder

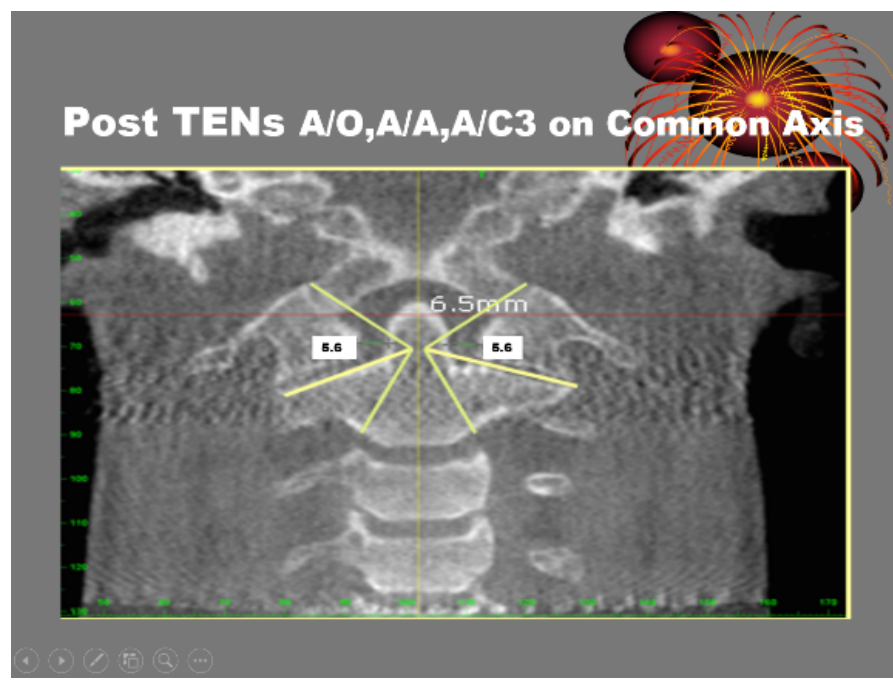

Figure 13. Post TENs A/O,A/A, A/C3 on common Axis

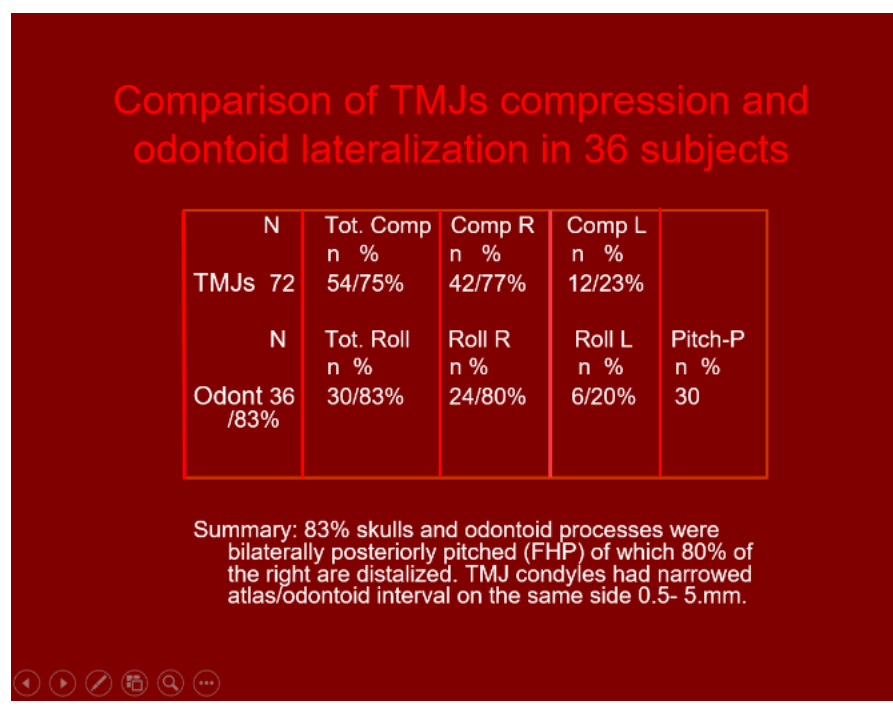

Figure 14. Comparison of TMJ compression and odontoid lateralization

\section{Nerve mediated TENS V,VII,XI,C 1}

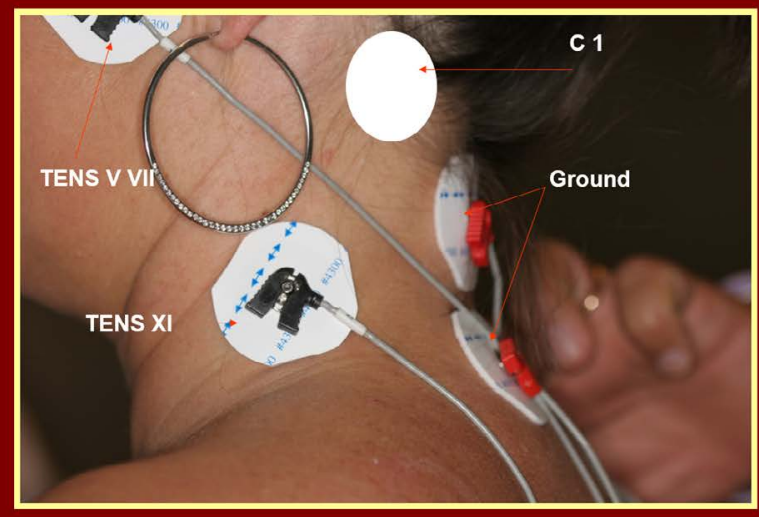

Figure 15. Nerve mediated TENS

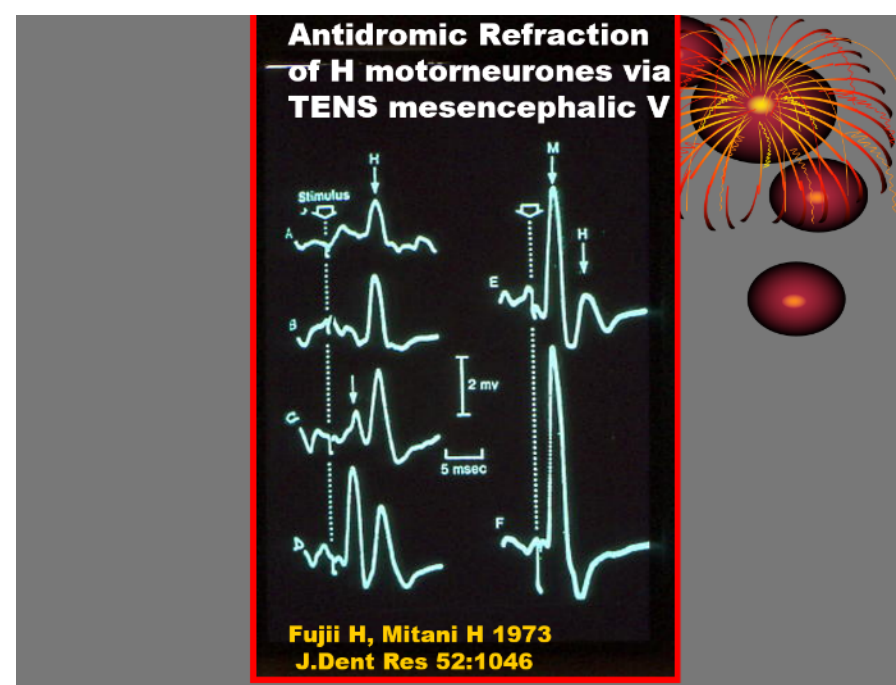

Figure 16. Antidromic refraction of $\mathrm{H}$ motorneurones via TENS mesencephalic V

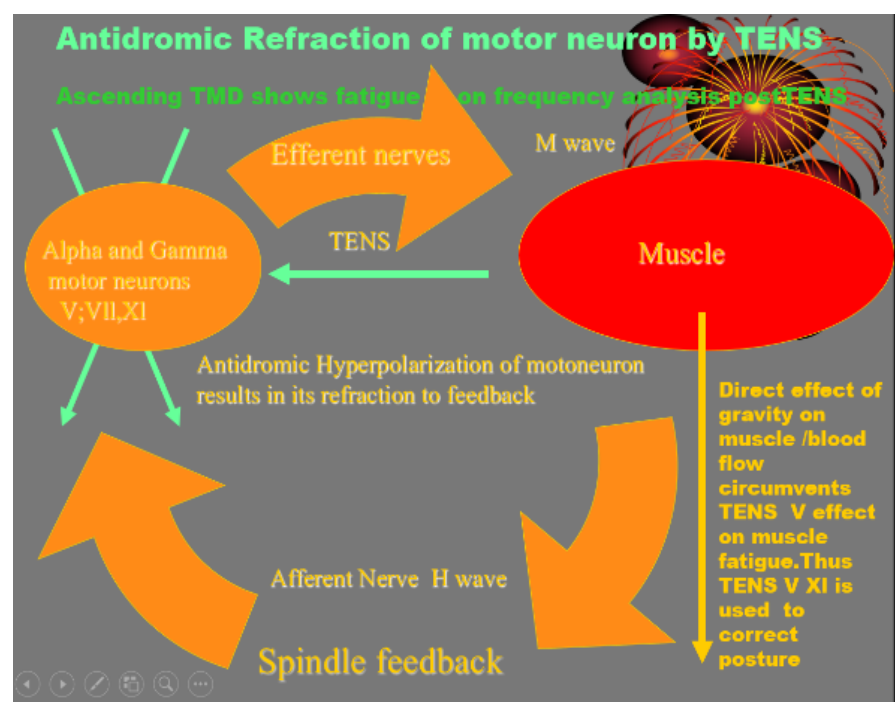

Figure 17. Antidromic refraction of motor neuron by TENS

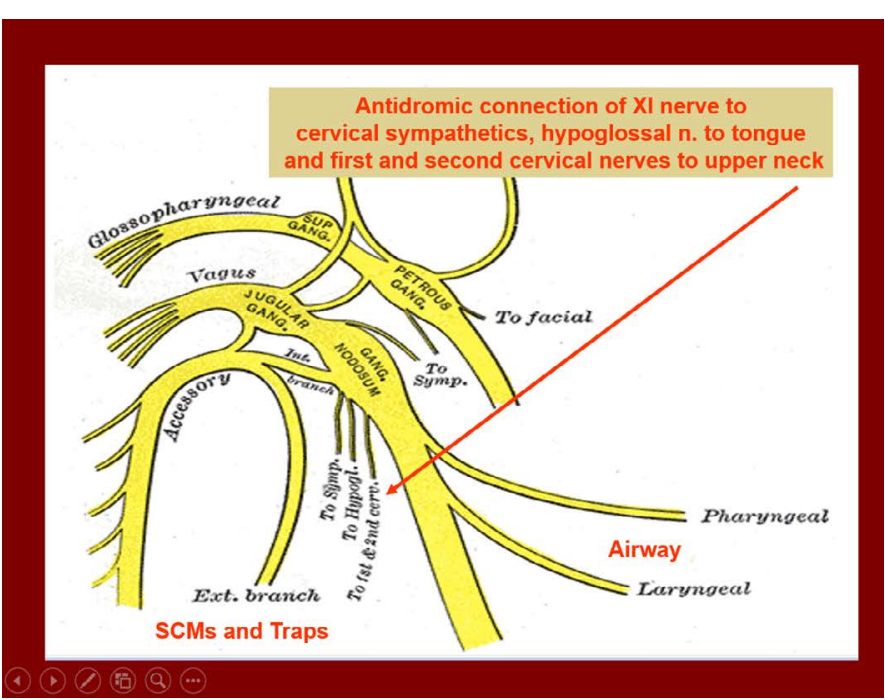

Figure 18. Antidromic connection 
Thomas NR (2019) The Relationship between the Dental Occlusion, Upper Cervical spine and Temporomandibular Joints prior to and following TENS treatment in thirty six patients suffering from Temporomandibular Disorder

11) indicative of an ascending effect of cervical spine malalignment, nonconcentricity of TMJs, A/O joints and occlusal imbalance as indicated by referred displacement of $\mathrm{C} 3$ and $\mathrm{C} 4$ at the position of hyoid bone a fulcrum for jaw opener muscles. Contrast the latter comparing pre and post TENS effects on the CBCT concentricity of the A/O, A/A, $\mathrm{C} 3 / \mathrm{C} 4$ and TMJs (Figures 14 and 15) clearly demonstrating the TENS effect on cranial nerves via Vth, VII and XI (Figures 13,14,17 and 18) motorneurone via alpha and gamma motorneurone affected by TENS activated proprioceptor input to the mesencephalic nucleus $\mathrm{V}$ allowing the craniomandibular and sub occipital muscles of the spine and neck.

\section{Conclusion}

From the general evidence discussed in this paper it is acknowledged that the dental occlusion mediates proprioceptor information about the position and movement of the head and neck in space via the trigeminal nerve principally the mesencephalic root of the trigeminal nerve. It is important to appreciate that the respective periodontal ligament nerve fibers have been shown to pass uninterrupted through the root of the mesencephalic nucleus of the fifth nerve to the masticatory motor (neurones proc N Am Int dent Res 1970 and Thomas NR IX International Anat Cong St Petersburg 1970 Russia.) and thence to the vestibular centre in the medulla which primarily receives information about the position and movement of the head in space notably from the labyrinth in the middle ear and upper cervical spine [12]. This input serves to affect the descending pattern of posture by the occlusion to the vestibular nucleus [13-15]. The ascending pattern of posture arises via the ascending system below the $\mathrm{C} 3 / \mathrm{C} 4$ hyoid bone level as described in the article. Transcutaneous electrostimulation (TENS) of the Vth, VIIth and XI cranial nerves produces relaxation of the masticatory, facial and neck muscles by antidromic relaxation and hyperpolarisation of the alpha gamma motorneurone of the jaw elevator and depressor muscles of the head, jaw and neck via the mesencephalic proprioceptor nucleus of the trigeminal nerve and hence correction of the descending pattern using the dental orthotic. I made use of a previous article published in LVIADS Visions July 2009 which was clarified in the present paper. This results in concentricity and alignment of the atlanto occipital A/O, atlanto axial $\mathrm{A} / \mathrm{A}$ and $\mathrm{C} 3 / \mathrm{C} 4$ vertebrae in part previously described by Thomas [1-3].

\section{References}

1. Thomas NR (1990) The effect of Fatigue and TENS on the EMG Mean Power Frequency 1990 Front Oral Physiol. Basil Karger 7: 162-170.

2. Raman P (2010) Neurally mediated ULF-TENS to relax Cervical and Upper Thoracic musculature as an Aid to Obtaining Improved Cervical Posture and mandibular Posture Anthology IX ICCMO.

3. Fujii H, Mitani H (1973) Reflex responses of the masseter and temporal muscles in man. J Dent Res 52: 1046-1050. [Crossref]

4. Cooperman HN, Willard SB (1955) Studies of the Louchheim Collection of Skulls American Museum of Natural History, New York, NY.

5. Harvold EP, Chierici G, Vargervik K (1978) Experiments on the development of dental malocclusions. Am J Orthod 61: 38-44. [Crossref]

6. Thomas NR (1966) The Process and Mechanism of Tooth Eruption PhD thesis University of Bristol, UK.

7. Thomas NR (2018) Inhibition of Collagen Crosslinking Produces Significant Retardation of Impeded and Unimpeded Eruption Rates. Calif Dent Assoc 46: 497-504.

8. Bimler HP (1985) Cephalometric Analysis Part .1. J Clin Orthod 18: 501-553.

9. Guzay CM (1980) Quadrant Theorem: A viewable Biophysical Analysis of Prosthodontia, Orthodontia, TMJ Disorders. TMData Resources.

10. Thomas MR, Thomas NR (1997) Temporomandibular Joint Dysfunction and Treatment Anthology IV ICCMO pp. 169-184.

11. Zafar H (2000) Integrated jaw and neck function in man. Studies of mandibular and headneck movements during jaw opening-closing tasks. Swed Dent J Suppl p. 1-41. [Crossref]

12. Thomas NR (1970) IX International Congress St Petersburg Russia.Symp VI Morphology of the Maxillo Mandibular Apparatus "Morphological and Physiological Studies of Mastication.

13. Cooperman HN (1975) HIP plane of occlusion in oral diagnosis. Dent Surv 51: 60-62. [Crossref]

14. Thomas NR (1976) Collagen: Generator of the Eruptive Force. Darling AI, Poole DFG, Stack (Eds) 27th. Colston Symposium, University of Bristol. pp. 290-310.

15. Thomas NR, Duncan M (2018) NuCalm brainwave entrained masseter muscle relaxation compared with TENS transcutaneous electro-neural stimulation of the fifth and seventh cranial motor nerves. Oral Health Care.

Copyright: (C2019 Thomas NR. This is an open-access article distributed under the terms of the Creative Commons Attribution License, which permits unrestricted use, distribution, and reproduction in any medium, provided the original author and source are credited. 